\title{
Acknowledgement to Reviewers of Scientia Pharmaceutica in 2018
}

Scientia Pharmaceutica Editorial Office

MDPI, St. Alban-Anlage 66, 4052 Basel, Switzerland

Published: 24 January 2019

Rigorous peer-review is the corner-stone of high-quality academic publishing. The editorial team greatly appreciates the reviewers who contributed their knowledge and expertise to the journal's editorial process over the past 12 months. In 2018, a total of 51 papers were published in the journal, with a median time to first decision of 15 days and a median time to publication of 38 days. The editors would like to express their sincere gratitude to the following reviewers for their cooperation and dedication in 2018:

Alvarez-Suarez, José Miguel
Andrade Caceres, Rafael
Angelova, Angelina
Anraku, Makoto
Araniciu, Cătălin
Assari, Shervin
Azuma, Kazuo
Bączek, Katarzyna
Badawi, Mohamed
Balalaeva, Irina
Balasubramanian, Krishnan
Baraldi, Pier Giovanni
Barbaric, Monika
Barone, Maria Vittoria
Bartolini, Manuela
Bastarrachea, Luis J.
Bertelloni, Fabrizio
Berthelsen, Ragna
Bhattacharya, Supriyo
Boudewyns, Vanessa
Bracher, Franz
Bucar, Franz
Burgalassi, Susi
Burger, Michael
Bylka, Wiesława
Caballero, Julio
Caiazzo, Elisabetta

Cardia, Maria Cristina

Cardoso, Susana

Carev, Ivana

Catucci, Lucia

Cebolla, Vicente L.

Cerella, Claudia

Chereddy, Kiran Kumar

Chien, Shih-Chang

Chiou, Chun-Tang

Cho, Eunae

Choudhary, Kumari Sonal

Christoforides, Elias

Cieslik, Wioleta

Corvigno, Sara

Cunningham-Rundles, Susanna

Dangol, Manita

De Jonghe, Steven

De Paula, Haroldo César Beserra

De Ruyck, Jérôme

De Vita, Valerio

De Wit, Maryna

De Zordi, Nicola

Deb, Subrata

Delorme, Vincent

DeLouise, Lisa A.

Ding, Wendu

Dkhar, Hedwin Kitdorlang 
Doi, Hirokazu

Dolowy, Malgorzata

Donadio, Carlo

Duran Lobato, Matilde

Ecke, Thorsten

Ecker, Gerhard

Edafiogho, Ivan

Egea, Marcos

Fasinu, Pius S.

Filannino, Pasquale

Fratini, Filippo

Froelich, Anna

Galeazzi, Roberta

Gallegos Monterrosa, Ramses

George Parsons, Kimberly Suzanne

Geronès, Ferran Feixas

Giner Pons, Rosa M.

González Barcia, Miguel

Goodman, Richard E.

Grant, George

Gumieniczek, Anna

Hamman, Joias

Hanganu, Daniela

Hartmann, Rolf

Hempel, Georg

Hinault, Thomas

Ho, Yi-Cheng

Houzé, Pascal

Imai, Toshio

Inngjerdingen, Marit

Islam, M. Nurul

Isola, Gaetano

Ita, Kevin

Jaradat, Nidal

Jelonek, Katarzyna

Ji, Tianjiao

Jones, Julie

Jones, Nicola

Juang, Shin-Hun

Juliano, Claudia

Kabała-Dzik, Agata

Kato, Takamitsu A.

Kawakami, Hiroshi
Kazakova, Oxana B.

Kevadiya, Bhaveah

Kipper, Karin

Komori, Teruhisa

Kowalczewski, Przemysław

$\mathrm{Ku}$, Seockmo

Kulkarni, Shashank

Lesyk, Roman B.

Li, Fuyang

Li, Lianbo

Lim, Beong Ou

Liu, Cheuk Lun

Liu, Rongming

Liu, Zhixia

Lugo-Cervantes, Eugenia

Luís, Ângelo

Lupien, Andréanne

Määttä, Sara

Madreiter-Sokolowski, Corina T.

Márialigeti, Károly

Matilla, Miguel

Michalska, Anna

Miroslaw, Barbara

Moreira, Vânia

Muceniece, Ruta

Mulinari, Shai

Naik, Shivangi

Nakonieczna, Joanna

Nayak, Shivananda

Nesterkina, Mariia

Nishi, Kosuke

Nounou, Mohamed Ismail

Ntie-Kang, Fidele

Nycz, Jacek E

Oćwieja, Magdalena

Otsuka, Yuzuru

Padrela, Luis

Panagiotidis, Mihalis

Papadimitriou, Konstantinos

Park, Hyunjin

Pedraza-Chaverri, Jose

Peltonen, Leena

Periyasamy, Palsamy 
Peterson, Eliza J.R.

Peterson, Larryn

Pinto, Mafalda

Pipan, Barbara

Przybyłek, Maciej

Quan, Zhe-Shan

Rajesh Lenin, Raji

Ranzato, Elia

Rizzuti, Bruno

Rosado, Catarina

Rosales, Carlos

Rubiolo, Patrizia

Ruggeri, Francesco

Russu, Wade

Sadkowski, Tomasz

Salaheen, Serajus

Santamaria, Rita

Saurav, Kumar

Schempp, Christoph M.

Schulze, Johannes

Sebastian, Aimy

Serradji, Nawal

Seto, Shigeki

Shakhtshneider, Tatyana P.

Shen, Chwan-Li

Shimpi, Manishkumar R.

Shults, Elvira E.

Siewert, Bianka

Sims, Ian M.

Sinha, Abhijeet

Spitzer, Martin

Sreerama, Subramanya
Starek, Małgorzata

Starostik, Petr

Starowicz, Małgorzata

Stephen, Michael Rajesh

Stuppner, Hermann

Sugawara, Akihiro

Szakonyi, Zsolt

Takaki, Manabu

Takano, Yu

Toiu, Anca

Tsuchiya, Yuichi

Ukrainets, Igor V.

Vanet, Anne

Varga, Zoltan

Vasile, Francesca

Vaz, Josiana

Venkatadri, Rajkumar

Victor, Bruno

Von Wright, Atte

Wang, Chao-Min

Wang, Jian-Wen

Wang, Sheng-Fan

Wang, Tong-Hong

Wang, Xiaoxing

Wang, Ying

Wojciezsyńska, Danuta

Wojtyczka, Robert

Yang, Yue

Yurovskaya, Marina A.

Zamyatnin, Jr., Andrey A.

Zhang, Hui 